\title{
DIE AWARISCH-ITALISCHEN BEZIEHUNGEN AUS GOLDSCHMIEDETECHNISCHER SICHT: EIN VORBERICHT
}

\author{
BIRGIT BÜHLER \\ Vienna Institute for Archaeological Science (VIAS) \\ 1 Franz-Klein-Gasse, A-1190 Wien, Österreich \\ birgit.buehler@univie.ac.at
}

\begin{abstract}
The technological choices made in the production process of a particular object can provide valuable clues regarding the identity and background of the goldsmith who made it. For example, true repoussé ("Treibziseliertechnik") was an uncommon technique throughout the entire Avar Period, although it occurs on a number of high-quality items of metalwork, which may be associated - for typological, stylistic and/or technological reasons - with "Byzantine" culture. Although the importance of connections between "Byzantine" and "Avar" culture is already well known, the mechanisms of cultural and technological transfer, as well as the regions where such transfer took place, are not yet clear. The aim of this paper is to demonstrate, with the help of two case studies, how a combination of technological criteria with an innovative, detailed typology of plant ornament may contribute to resolving these questions. The results of this preliminary study suggest that "Italo-Byzantine" workshop traditions may have played an important role in this context.
\end{abstract}

Keywords: Avar archaeology, early medieval metalworking techniques, early medieval plant-ornament, Nagyszentmiklós treasure

\section{ALLGEMEINES}

Die Auswahl der für die Anfertigung eines bestimmten Metallobjektes verwendeten Techniken, Arbeitsschritte und Werkzeugtypen ist nur zum Teil durch technische Vorgaben bedingt: In vielen Fällen stehen innerhalb des „Rahmenwerks“ herstellungstechnischer Notwendigkeiten eine Reihe von Alternativen zur Verfügung. Welche der möglichen Techniken, Arbeitsschritte und Werkzeugtypen im Einzelfall verwendet werden, hängt einerseits vom Können bzw. der Ausbildung sowie den persönlichen Präferenzen des betreffenden Handwerkers ab, wobei auch handwerkliche Traditionen (z. B. einer einzelnen Werkstatt, eines lokalen, regionalen oder überregionalen Werkstattkreises sowie einer bestimmten Epoche) eine Rolle spielen können. Andererseits beeinflussen selbstverständlich auch die Vorgaben des Auftraggebers (z. B. hinsichtlich der dargestellten Motive, des Stils, der verwendeten Techniken und des Materials bzw. der Materialmenge) den Herstellungsvorgang. Folglich enthalten nicht nur Form und Verzierung, sondern auch die technischen Entscheidungen, die im Zuge des Herstellungsprozesses eines bestimmten Metallobjektes getroffen worden sind, Informationen sowohl über den Auftraggeber als auch über den Hersteller sowie deren Umfeld.

Eine Synthese der Ergebnisse der typologischen, stilistischen und herstellungstechnischen Untersuchungen kann im Idealfall eine Zuordnung zu bestimmten Werkstätten sowie lokalen, regionalen oder überregionalen Werkstattkreisen ermöglichen: Somit ist es beispielsweise möglich, Hinweise auf Art und Ausmaß der „Beziehungen“ zwischen dem awarischen Herrschaftsbereich und dessen Nachbarregionen zu erhalten. Wichtig wäre es in diesem Zusammenhang, bisherige Überlegungen und Erklärungsmodelle bezüglich der Mechanismen des Transfers von 
Formen, Motiven, Techniken etc. zu überprüfen (d. h. im Hinblick auf die Mobilität von Personen, Objekten und Gebräuchen bzw. „Modeerscheinungen“).

Am „multikulturellen Charakter“ der awarischen Kultur und an der Rolle, die „byzantinische“ Kulturkontakte bei ihrer Herausbildung im Karpatenbecken gespielt haben, besteht nach der intensiven Forschungstätigkeit der letzten Jahrzehnte zu diesem Thema kein Zweifel mehr. Die Beziehungen zum langobardenzeitlichen Italien dürften in diesem Zusammenhang eine wichtige Rolle gespielt haben: Dies zeigen mehrere Objektgruppen, die mit großer Wahrscheinlichkeit als Importe bzw. Handelsware angesprochen (z. B. in Italien geprägte Münzen, bestimmte Typen von Bronze- und Glasgefäßen, gegossene Gürtelgarnituren vom „alpinen“ bzw. wohl vielmehr „norditalischen“ Typ, eventuell auch bestimmte Tauschierarbeiten) werden können”.

Die Werkstattfunde aus der Crypta Balbi (Rom) ${ }^{2}$ zeigen, wie vielfältig die Verarbeitung von Bunt- und Edelmetallen in italo-byzantinischen Werkstätten des 7. Jahrhunderts gewesen ist: Dies trifft sowohl bezüglich der verwendeten Materialien, Herstellungs- und Verzierungstechniken als auch in ikonographischer Hinsicht zu. Insgesamt entsteht der Eindruck, dass in italo-byzantinischen Goldschmiedewerkstätten noch im 7. Jahrhundert Traditionen des spätantiken Feinschmiedehandwerks lebendig waren: Hierfür sprechen sowohl ornamentale Details (insbesondere des pflanzlichen Dekors) als auch herstellungstechnische Aspekte. Ein Schwerpunkt der metallverarbeitenden Werkstätten in der Crypta Balbi dürfte die Herstellung von dekorativen Gürtelschnallen und -garnituren, aber auch anderer Schmuckstücke und Ausrüstungsgegenstände, wie z. B. Bestandteile des Pferdezaumzeugs und Sattelbeschläge gewesen sein. Die Funde aus der Crypta Balbi enthalten auch Model und Halbfertigprodukte, die mit Halbpalmettenornamentik im Stil der Gruppe „Stabio-Beromünster-Utrecht“ verziert sind. Die italische Herkunft dieser Stilgruppe wurde bereits vor längerer Zeit postuliert ${ }^{3}$ und wird durch die Werkstattfunde aus der Crypta Balbi bestätigt. Aufgrund von in der Crypta Balbi gefundenen Modeln und Halbfabrikaten ist bei dieser Objektgruppe ein Werkstattzusammenhang mit goldenen Fertigprodukten aus reich ausgestatteten langobardischen Gräbern anzunehmen ${ }^{4}$. Daher ist davon auszugehen, dass die Werkstätten der Crypta Balbi im 7. Jahrhundert Feinschmiedearbeiten gehobener Qualität, sowohl für „römisch-byzantinische“ als auch für „langobardische“ Eliten, produziert haben ${ }^{5}$.

Im Hinblick auf goldene awarische Treibziselierarbeiten sind vor allem die aus Goldblech gefertigten Sattel- und Zaumzeugbeschläge aus langobardischen Gräbern von besonderem Interesse: Somit ist die Gestaltung plastischen Dekors aus Blechen aus Edelmetall durch Treibziselierung, Punzierung und Pressblechtechnik in italischen Goldschmiedewerkstätten des 7. Jahrhunderts nachgewiesen. Anzumerken ist, dass bei den betreffenden Objekten in manchen Fällen eine Kombination der Pressblechtechnik mit Punzierung und Treibziselierung anzunehmen ist: Dies betrifft einerseits die Gestaltung von Details, andererseits die Präzisierung der Konturen des plastisch gestalteten Dekors.

Innerhalb des awarischen Siedlungsgebiets sind Treibziselierarbeiten ${ }^{6}$ im 7.-8. Jahrhundert nur vereinzelt belegt. Die betreffenden Exemplare bilden eine kleine Gruppe von aus Goldblech gefertigten Produkten gehobener Qualität: Letztere beinhalten sowohl Gürtelgarnituren des 7. und 8. Jahrhunderts (z. B. Kunágota, Igar, Brestovac, Mátészalka, Weiden am See), als auch andere Objekte wie Agraffen (z. B. Dunapataj) und Gefäße: Denn an der Spitze dieser Gruppe steht - bezüglich der herstellungstechnischen und künstlerischen Qualität - der Goldschatz von Nagyszentmiklós.

In Italien gefundene Feinschmiedearbeiten des 7. Jahrhunderts könnten dazu beitragen, die herstellungstechnische „Lücke“ zu den relativ hochwertigen, im awarischen Siedlungsgebiet gefundenen Treibziselierarbeiten aus Edelmetall zu schließen. In diesem Zusammenhang sind vor allem mehrere goldene Sattelbeschläge ${ }^{7}$ aus langobardischen Grabfunden (z. B. Castel Trosino Grab 119, Nocera Umbra Grab 5), die aus Silberblech gefertigten

${ }^{1}$ Vgl. zusammenfassend: BÁLINT 2010, 617-620; GARAM: 2002, 98-99; A. Kiss: Das awarenzeitlich gepidische Gräberfeld von Kölked-Feketekapu A. Monographien zur Frühgeschichte und Mittelalterarchäologie 2. Innsbruck 1996, 300-302. Weiters: Cs. BÁLINT: The Avars, Byzantium and Italy: A study in chorology and cultural history. VAH 31. Budapest 2019 (im Druck).

${ }^{2}$ Roma dall'antichità al medioevo. Archeologia e storia nel Museo Nazionale Romano Crypta Balbi. A cura di M. S. Arena, P. Delogu, L. Paroli, M. Ricci, L. Sagui, L. Vendittelli. Milano 2001.
${ }^{3}$ G. HASELOFF: Das Warnebertus-Reliquiar im Stiftsschatz von Beromünster. HelvA 11 (1984) 195-217.

${ }^{4}$ RICCI 2012, 1-16.

${ }^{5}$ N. ChristiE: Byzantines, Goths and Lombards in Italy: Jewellery, dress and cultural interactions. In: Intelligible Beauty: Recent Research on Byzantine Jewellery. Eds: Ch. Entwistle, N. Adams. London 2010, 113-122.

${ }^{6}$ Vgl. z. B. BÜHLER 2014.

${ }^{7}$ RiCCI 2012, 1-16. 
Schwertscheidenbeschläge mit Tiermotiv und pflanzlichem Dekor aus Morrione Grab $102^{8}$ sowie die sog. „AgilulfPlatte“9 aus vergoldetem Bronzeblech zu nennen. Hervorzuheben ist in diesem Zusammenhang, dass sowohl herstellungstechnische als auch stilistische Übereinstimmungen zwischen den Funden aus Italien und den im awarischen Siedlungsgebiet gefundenen, Treibziselierarbeiten aus Goldblech festgestellt werden können. Hierzu gehören auch Eigenheiten bezüglich der Strukturierung von Oberflächen, wie z. B. die flächige Punktierung des treibziselierten Dekors mittels besonders feiner Perl- oder Körnerpunzen, wie sie unter anderem auch für Treibziselierarbeiten der „Stäbchenrankengruppe“ des Schatzfundes von Nagyszentmiklós kennzeichnend sind (s. u., Fallbeispiel 2).

Die weite Verbreitung unterschiedlichster Varianten pflanzlicher Ornamentik in den Kern- und Randgebieten der byzantinischen Welt macht sie zu einem geeigneten Ansatzpunkt für die Untersuchung von frühmittelalterlichen Kulturbeziehungen ${ }^{10}$. Die Voraussetzung für eine solche Studie ist eine exakte, nachvollziehbare Terminologie sowie eine verfeinerte, typologische Gliederung pflanzlicher Ornamente, die in weiterer Folge kulturhistorisch interpretiert werden kann. Eine vergleichende Analyse pflanzlicher Ornamente kann auf unterschiedlichen Ebenen der Komplexität durchgeführt werden: Es kann durchaus sinnvoll sein, zunächst ein einzelnes Zierelement (z. B. tropfenförmige Blättchen; s. u.) herauszugreifen und dessen Verbreitung in unterschiedlichen Varianten bzw. unterschiedlicher herstellungstechnischer Ausführung in den betreffenden Kulturräumen zu studieren bzw. zu vergleichen. An anderer Stelle wurde darauf hingewiesen ${ }^{11}$, dass bestimmte Varianten awarischen Pflanzendekors, insbesondere die „Stäbchenranken- und Lilienzier“, aber auch die Ranken- und Halbpalmettenzier, aufgrund von Kontakten zum „byzantinischen“ Kulturraum entstanden sein dürften. Ebenda wurde auch bereits angemerkt, dass Kulturbeziehungen nach Italien in diesem Zusammenhang eine wichtige Rolle spielen könnten. Eine erneute Durchsicht des Fundmaterials, unter besonderer Berücksichtigung der Feinbearbeitungstechniken, könnte nunmehr eine Präzisierung des Herkunftsgebietes dieser Einflüsse ermöglichen, wobei in diesem Zusammenhang die Kombination einer Feintypologie pflanzlicher Ornamentik mit herstellungstechnischen Kriterien von besonderer Bedeutung ist.

Ein guter Ansatzpunkt für die Identifizierung kunsthandwerklicher Traditionen in der Goldschmiedetechnik ist die Strukturierung von Oberflächen: Hierbei ist zunächst zwischen einer Strukturierung des Hintergrundes und einer Strukturierung des Motivs zu unterscheiden, wobei Letztere meist vielfältiger und differenzierter ist. Das Verhältnis zwischen Dekor und Hintergrund stellt ein wichtiges stilistisches Merkmal dar. Jedoch existieren in vielen Fällen, mehrere herstellungstechnische Lösungen für einen bestimmten Effekt. Aus feinschmiedetechnischer Sicht gibt es z. B. eine Vielfalt von Möglichkeiten zur Strukturierung von Metalloberflächen. Von Interesse sind in diesem Zusammenhang zunächst die, jeweils für die Feinbearbeitung eingesetzten Werkzeugtypen und ihre Verwendung, unter besonderer Berücksichtigung bestimmter Eigenheiten in der Gestaltung, sowie der Kombination bestimmter Motive, Feinbearbeitungstechniken und Werkzeugtypen. Die jeweils gewählte Kombination von Motiven, Bearbeitungstechniken und Werkzeugtypen erlaubt Rückschlüsse bezüglich kunsthandwerklicher Traditionen und kann daher Aussagen zu Kulturkontakten ermöglichen.

Auch im Zuge der Untersuchung des Schatzfundes von Nagyszentmiklós hat ein Vergleich der zur Strukturierung von Flächen (im Bereich des Hintergrundes bzw. des Motivs) verwendeten Punzentypen wesentlich zur Feingliederung des Fundkomplexes beigetragen ${ }^{12}$. Durch die Verbindung einer Feintypologie pflanzlicher Ornamentik mit herstellungstechnischen Kriterien (z. B. zur Strukturierung bestimmter Motive verwendete Punzentypen) ergeben sich jedoch zusätzliche Möglichkeiten der historischen Interpretation (z. B. im Hinblick auf Kulturbeziehungen $)^{13}$, wie hier am Beispiel der tropfenförmigen Blättchen gezeigt werden soll (s. u., Fallbeispiel 2).

${ }^{8}$ V. CEGLiA: The necropolis of Campochiaro in Italy. In Rome and the Barbarians. Exhibition Catalogue, Palazzo Grassi. Venice 2008, 470, Cat. No. V17.

${ }^{9}$ Brogiolo-Chavarría Arnau 2007, 55-56, Fig. 1.1.15.

${ }^{10}$ Zur pflanzlichen Ornamentik der Spätawarenzeit vgl zuletzt: G. SzENTHE: Vegetal ornaments in the Late Avar decorative art. DissArch 3/1 (2013) 303-320; sowie zur „Lilienzier“: M. M. E. SCHMID: Das Gräberfeld von Rákóczifalva in Zentralungarn und die Chronologie des spätawarenzeitlichen Fundmaterials. UPA 272. Bonn 2015.
${ }^{11}$ BüHLER 2014; B. BüHLER: Der Schatz von Brestovac (Kroatien) und seine kulturellen Beziehungen unter besonderer Berücksichtigung der technologischen Aspekte. Diss., Universität Wien 2002; B. BÜHLER: Ranken und Palmetten. Studien zu pflanzlichen Ornamenten im Frühmittelalter. AÖ 11/2 (2000) 60-72.

${ }^{12}$ BÜHLER-Freiberger 2018, Teil 1, 23-201

${ }^{13}$ Eine umfangreichere Arbeit zu diesem Thema ist in Vorbereitung. 


\section{FALLBEISPIEL 1: STRUKTURIERUNG DES HINTERGRUNDES MITTELS RINGFÖRMIGER PUNZIERUNGEN}

Gravierter, pflanzlicher Dekor auf flächig ringpunziertem Hintergrund ist für spätawarische Gürtelbeschläge besonders charakteristisch: Diese Variante pflanzlichen Dekors dürfte zunächst, in der ersten Hälfte des 8. Jahrhunderts, vorwiegend in Kombination mit anderen Motiven und Feinbearbeitungstechniken zur Verzierung gegossenen Gürtelschmucks verwendet worden sein. In der Spätawarenzeit III (letztes Drittel 8. bzw. Anfang 9. Jahrhundert) wurde diese Art des Dekors jedoch zunehmend als einzige Verzierungstechnik des betreffenden Gürtelbeschlags eingesetzt ${ }^{14}$. Aber auch gegossene spätawarische Beschläge, die ein mitgegossenes Relief und einen flächendeckend ringpunzierten Hintergrund aufweisen, sind in der Spätawarenzeit II-III (zweites und letztes Drittel des 8., eventuell auch Anfang des 9. Jahrhunderts) gebräuchlich und außerdem mit verschiedenartigsten Motiven verziert, wobei Motive „mediterraner“ Herkunft besonders häufig vertreten sein dürften.

Obwohl in beiden Fällen eine Strukturierung des Hintergrundes mittels eines Ringpunzens vorliegt, ist es - sowohl aus herstellungstechnischer als auch aus stilistischer Sicht - wichtig, zwischen nicht plastisch (d. h. linear) gestaltetem und plastisch gearbeitetem Dekor mit ringpunziertem Hintergrund auf Bunt- und Edelmetallobjekten zu differenzieren. Nicht-plastisch bzw. linear gestalteter (d. h. gravierter oder geschroteter) Dekor mit flächig ringpunziertem Hintergrund kann sowohl zur Verzierung von gegossenen als auch von, aus Blech gefertigten, Produkten aus Bunt- und Edelmetall verwendet werden. Diese Variante steht hier im Zentrum des Interesses (vgl. Abb. 1). Bei Objekten mit plastisch gestaltetem Dekor auf ringpunziertem Hintergrund (vgl. Abb. 2) muss man wiederum zwischen gegossenen und getriebenen Produkten unterscheiden. Bei gegossenen Objekten wurde der plastisch gestaltete Dekor mitgegossen und durch Punzierung (Reliefgrund = Ringpunzen; Dekor = Dreieckspunzen, Perl-/ Körnerpunzen etc.) nachbearbeitet (Beispiel: Riemenzunge mit Vogelmotiv aus Mikulčice ${ }^{15}$ ), bei Exemplaren aus Blech formte man den plastisch gestalteten Dekor durch Treibziselierung oder in Pressblechtechnik und punzierte den Reliefgrund mittels eines Ringpunzens (Beispiel: Rankenzier im Bereich der Mündung des Kruges Nr. 2 von Nagyszentmiklós ${ }^{16}$ ).

Zu beachten ist auch, dass ein optisch ähnlicher Effekt ebenso mittels flächiger „Punktierung“ des Hintergrundes erzielt werden kann, wobei anstelle von Ringpunzen Feinwerkzeuge mit annähernd runder Arbeitskante (Perlpunzen, Körnerpunzen) verwendet werden: Als Beispiel kann der nicht-plastisch gestaltete, pflanzliche Dekor auf dem Beschlag einer Silberschnalle aus dem spätantiken Schatzfund von Desana ${ }^{17}$ angeführt werden. Es ist anzunehmen, dass beide Varianten (d. h. nicht-plastisch gestalteter Dekor mit ringpunziertem bzw. „punktiertem“ Hintergrund) ursprünglich aus dem römischen Feinschmiedehandwerk stammten und in byzantinischer Zeit weiter verwendet worden sind ${ }^{18}$.

Grundsätzlich dürfte die Variante mit nicht plastisch gestaltetem Dekor auf ringpunziertem Hintergrund, nicht nur im awarischen Siedlungsgebiet, sondern auch im Mittelmeerraum bei Weitem die gebräuchlichste der bereits genannten Varianten mit ringpunziertem Hintergrund gewesen sein. Zahlreiche Beispiele für diese Verzierungstechnik finden sich auf byzantinischen Bronzeschnallen mit u-förmigem Beschläg (Typ F 5 nach SchulzeDörrlamm $)^{19}$ : Allerdings ist die überwiegende Mehrzahl der Exemplare dieses Typs ohne genauen Fundort. Dies ist besonders bedauerlich, da eine beträchtliche Anzahl der Schnallen dieses Typs nicht nur feinschmiedetechnische, sondern auch stilistische Übereinstimmungen (z. B. gelappte, halbpalmettenartige Blattmotive) mit den Vergleichsbeispielen aus dem awarischen Siedlungsgebiet aufweist. Von besonderem Interesse ist ein goldenes Exemplar dieses Typs (leider ebenfalls ohne Provenienz), dessen Schnalle mit eingravierter Halbpalmettenzier auf ringpunziertem Hintergrund versehen ist ${ }^{20}$.

Im Hinblick auf eine mögliche „,italische“ Herkunft des spätawarischen, nicht plastisch gestalteten pflanzlichen Dekors auf ringpunziertem Hintergrund stellt das Goldkreuz aus dem sog. „Schatz der Theodelinde“ im Dom von Monza einen Schlüsselfund dar. Die Rückseite dieses Schmuckstücks, das Papst Gregor der Große im Jahr 603 dem langobardischen Königssohn Adaloald zur Taufe geschenkt haben soll, ist mit pflanzlichem Dekor verziert, der

\footnotetext{
${ }^{14}$ GARAM 2002, 105-110.

${ }^{15}$ BÜHLER 2014, Taf. 40/1-3.

${ }^{16}$ BÜHLER-FreIBERgER 2018 , Teil 2, Taf. 35.

${ }^{17}$ Brogiolo-Chavarría Arnau 2007, 187-188, Fig. 3.5f.

${ }^{18}$ In diesem Zusammenhang ist es bemerkenswert, dass die Strukturierung des Hintergrundes mittels ringförmiger Punzierun-
}

gen bei orientalischen Goldschmiedearbeiten erst im 8. Jahrhundert belegt ist; vgl. hierzu: BÁLINT 2010, 186-201.

${ }^{19}$ SCHULZE-DÖRRLAMM 2009, 149-155.

${ }^{20}$ SCHUlze-DörRlamm 2009, 164-167, Abb. 73. 


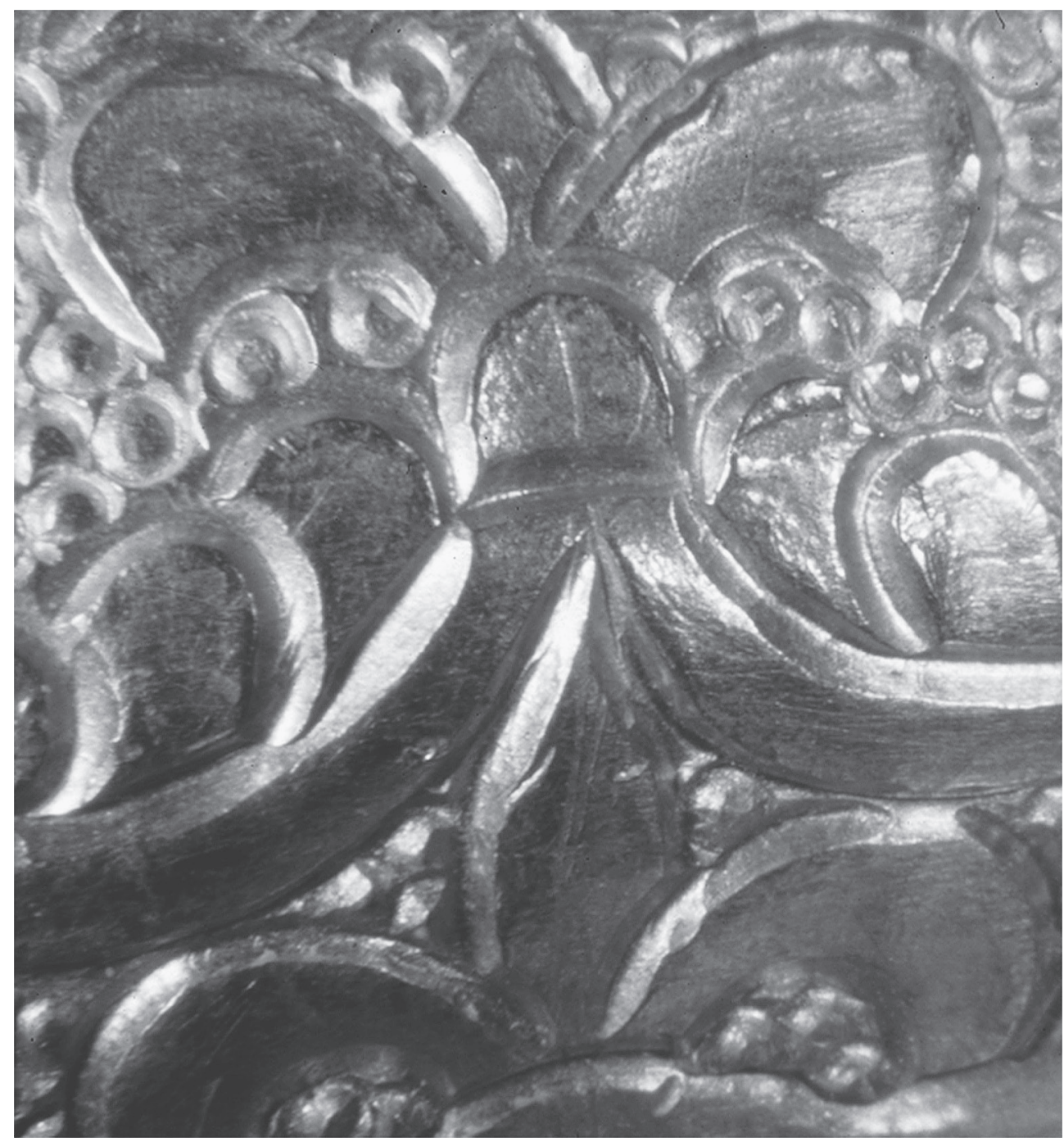

Abb. 1. Kunsthistorisches Museum Wien, Antikensammlung, Inv. Nr. VII B 28, Goldschatz von Nagyszentmiklós (heute: Sânnicolau Mare, Rumänien), Krug Nr. 6 (nach Hampel). Lichtmikroskopisches Detail des Fussbereichs: geschroteter, floralgeometrischer Dekor mit ringpunziertem Hintergrund sowie tropfenförmigen Blättchen der Variante 3 (Foto: Birgit Bühler)

- sowohl in herstellungstechnischer als auch in stilistisch-motivischer Hinsicht - hervorragende Parallelen im awarischen Fundmaterial besitzt ${ }^{21}$ : Im Zentrum der Rückseite befindet sich ein kreuzförmiges Motiv (= „Blattkreuzmotiv“) innerhalb eines runden Medaillons. Das „Blattkreuz“ besteht aus vier „lilienartigen“ Dreiblattmotiven ( = Blattkelch, gebildet von zwei spitzen Blättchen dazwischen sitzt ein zentrales, tropfen- bis lanzettförmiges Blättchen), die durch ein zentrales Kreispunktmotiv, zusammengehalten werden. Jeder der vier Kreuzarme ist mit einem „bäumchenartigen“ Motiv verziert, das jeweils aus drei Halbpalmettenpaaren zusammengesetzt ist: Die Halbpalmetten sind jeweils dreiblättrig und erinnern bezüglich ihrer „gelappten“ Gestaltung an identisch gearbeitete, pflanzliche Ornamente auf spätawarischem Gürtelzubehör.

Von besonderem Interesse sind jedoch Vergleichsbeispiele - sowohl in goldschmiedetechnischer als auch motivisch-stilistischer Hinsicht - aus dem Schatzfund von Nagyszentmiklós: Der Fuß des Kruges Nr. $7^{22}$ ist mit einem repetierenden, „bogenfriesartigen“ Muster verziert, das aus „lilienartigen“ Dreiblattmotiven besteht. An der 


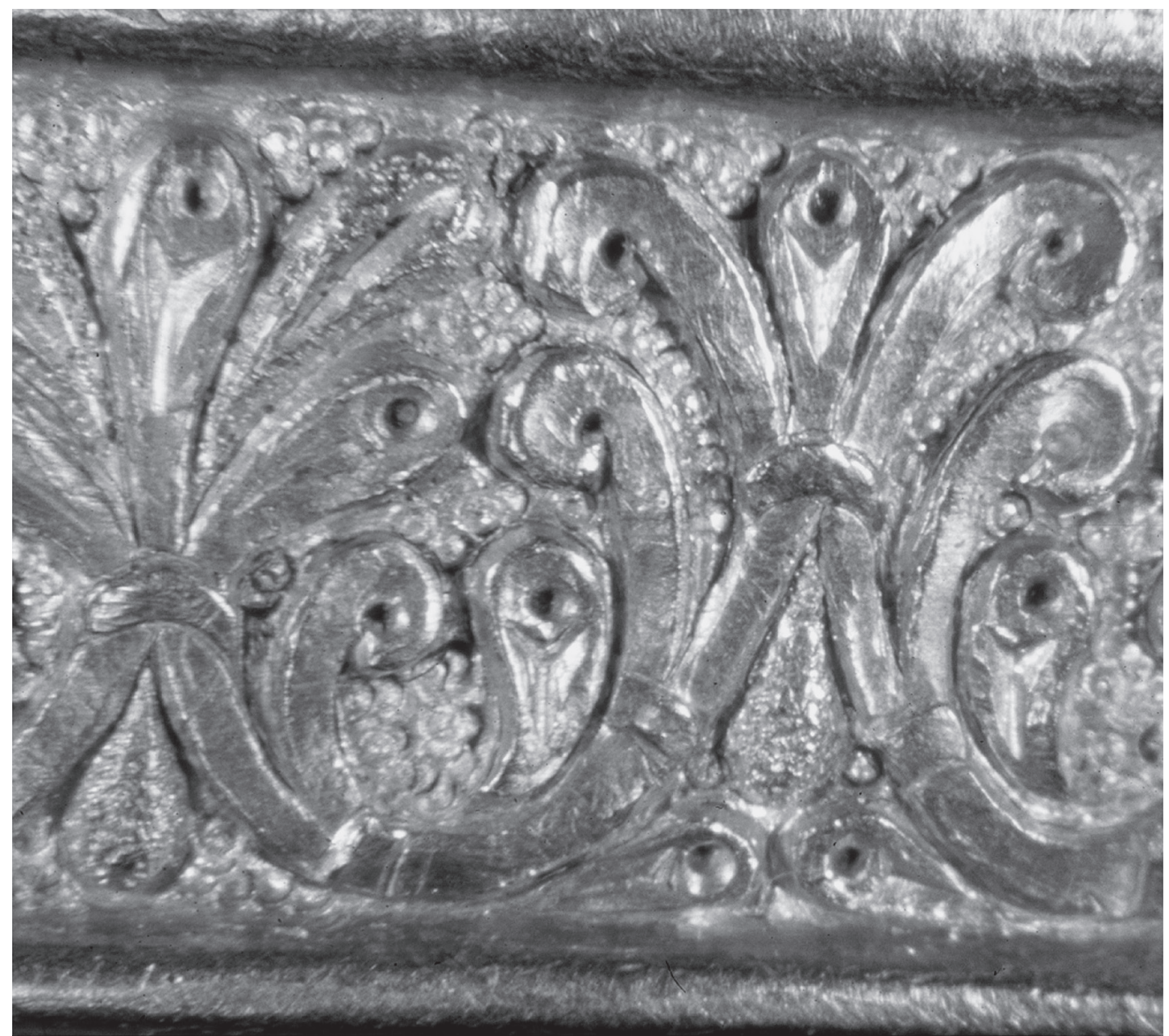

Abb. 2. Kunsthistorisches Museum Wien, Antikensammlung, Inv. Nr. VII B 10, Goldschatz von Nagyszentmiklós (heute: Sânnicolau Mare, Rumänien), ,gehörnte Löwenschale“ Nr. 13 (nach Hampel).

Lichtmikroskopisches Detail des Schalenrandes: treibziselierte „Stäbchenrankenzier“ mit ringpunziertem Hintergrund und tropfenförmigen Blättchen der Varianten 2, 4a \& 5 (Foto: Birgit Bühler)

Basis jedes dieser „lilienartigen“ Dreiblattmotive befindet sich ein Kreispunktmotiv, das wiederum jenem im Zentrum des „Blattkreuzmotivs“ auf dem Kreuz von Monza entspricht. Die Konturen des soeben beschriebenen, floralgeometrischen Dekors sind geschrotet und der Hintergrund mit feinen, ringförmigen Punzierungen bedeckt. Der floralgeometrische Dekor auf dem Fuß des Krugs Nr. 7 ist in derselben Technik gearbeitet wie der pflanzliche Dekor auf der Rückseite des Kreuzes aus dem Domschatz von Monza. Weitere Parallelen zum pflanzlichen Dekor des Kreuzes von Monza - nicht nur bezüglich der verwendeten Technik, sondern auch aufgrund der gelappten Blätter der Rankenzier - finden sich im Mündungsbereich des Kruges Nr. $3^{23}$ sowie auf der Griffunterseite der Schale Nr. $8^{24}$ aus dem Schatzfund von Nagyszentmiklós.

Zudem ist anzumerken, dass (nicht plastisch gestalteter) Dekor mit gravierten oder geschroteten Konturen und ringpunziertem Hintergrund auf einer Reihe besonders hochwertiger Goldschmiedearbeiten, die dem (italo-) byzantinischen Kunsthandwerk nahestehen dürften, als ,zusätzliche Technik“ zur Gestaltung von ornamentalen Details auf der Rückseite oder an anderen, wenig beachteten Stellen des Objektes verwendet worden ist. Im Hinblick auf eventuelle awarisch-italische Beziehungen im Bereich des Goldschmiedehandwerks ist die aus Messing 
in Komposittechnik gefertigte, vergoldete Gürtelgarnitur von Hohenberg, bei der es sich nach F. Daim ${ }^{25}$ um ein italo-byzantinisches Produkt handeln dürfte, von besonderer Bedeutung: Auf Rahmen, Dorn und Scharnier der Schnalle der Gürtelgarnitur von Hohenberg ist jeweils gravierter pflanzlicher Dekor vorhanden. Es handelt sich um Rankenzier sowie um diverse einfache Blatt- und Blütenmotive. Zudem weisen alle Scharnierbeschläge der Gürtelgarnitur von Hohenberg, jeweils nur im Bereich des Scharniers, eingravierte, zweiblättrige Blütenmotive auf ringpunziertem Hintergrund als zusätzlichen Dekor auf.

Auch auf dem Scharnier des in Komposittechnik gefertigten Scharnierbeschlags von Szeged-Kiskundorozsma $^{26}$ aus vergoldetem Silber ist einfacher, pflanzlicher Dekor mit gravierten Konturen auf flächig ringpunziertem Hintergrund als zusätzliches Zierelement vorhanden. Wie bei der Gürtelgarnitur von Hohenberg, sind auch bei diesem Exemplar, neben anderen dekorativen Techniken, die für das (italo)-byzantinische Goldschmiedehandwerk charakteristisch sein dürften (z. B. Durchbruchsarbeit, Verwendung zweiteiliger Model, Perldraht und Granulation), einfache eingravierte Blütenmotive auf flächig ringpunziertem Hintergrund vertreten.

„Lilienartige“ Dreiblattmotive, die formal mit jenen auf dem Goldkreuz von Monza übereinstimmen, bilden auch das Grundelement der zentralen, durchbrochen gearbeiteten Cabochonfassung auf der Oberseite der goldenen Agraffe von Dunapataj (Ungarn) ${ }^{27}$ : Insgesamt ist die Agraffe von Dunapataj durch zahlreiche herstellungstechnische Besonderheiten, die auf einen Zusammenhang mit dem (italo-),,byzantinischen“ Kunsthandwerk hinweisen, gekennzeichnet. Besonders bemerkenswert ist die Vielfalt der miteinander kombinierten Techniken, die auch die hier diskutierte Variante pflanzlicher Ornamentik beinhaltet:

- Aus zahlreichen Einzelteilen aus Goldblech zusammengesetzt („Kompositbauweise“)

- Treibziseliertechnik

- Durchbruchsarbeit

- Nicht plastisch gestalteter, pflanzlicher Dekor („Palmettenzier“) auf flächig ringpunziertem Hintergrund

- Cabochons mit nicht erhaltenen Glas- oder Edelsteineinlagen

- Perldraht

- Granulation

- Befestigung durch „Ösenschlaufen“

Die Cabochonfassung auf der Oberseite der Agraffe von Dunapataj ist von einer Reihe zweiteiliger, aus Goldblech gefertigter Komponenten annähernd halbrunder Form umgeben: Auf eine konische Basis wurde ein flach-halbkugeliges Goldblech, das mit einer gravierten Palmette auf einem Hintergrund aus sehr feinen, ringförmigen Punzierungen verziert ist, aufgelötet. Dieselben zweiteiligen, halbrunden Zierelemente finden sich auch am äußeren Rand, dazwischen befindet sich eine Reihe von Goldblechringen, die ebenfalls flächendeckend mit ringförmigen Punzierungen verziert worden sind. Letztere sind allerdings deutlich gröber als jene, die den Hintergrund der Palmettenzier bilden. Auch hier stellt der nicht plastisch gestaltete, pflanzliche Dekor auf flächig ringpunziertem Hintergrund lediglich eine zusätzliche Verzierungstechnik dar, die nur bei genauer Betrachtung des Objektes erkennbar ist: Es handelt sich um ein stilisiertes Blatt- bzw. Halbpalmettenmotiv, dessen Konturen vermutlich eingraviert worden sind. Bei dem hier zur Gestaltung des Hintergrundes verwendeten Ringpunzen dürfte es sich um ein auffallend feines Werkzeug gehandelt haben.

${ }^{25}$ F. DAIM: „Byzantinische“ Gürtelgarnituren des 8. Jahrhunderts. In: Die Awaren am Rand der byzantinischen Welt. Studien zu Diplomatie, Handel und Technologietransfer im Frühmittelalter. Hrsg.: F. Daim. Monographien zur Frühgeschichte und Mittelalterarchäologie 7. Innsbruck 2000, 77-204.

${ }^{26} \mathrm{Vgl}$. Abbildung auf dem Umschlag (v. a. Rückseite) des Jahresberichtes 2007 des Römisch Germanischen Zentralmuseums Mainz; Vgl. auch: F. DAIM-S. GreIFF-S. PATSCHER: KAISER mit Palm- zweigen. Ein byzantinischer Gürtelbeschlag aus Südungarn. JbRGZM 53/3 (2007) [2008] 77-79.

${ }^{27}$ F. DAIM-B. BÜHLER: Awaren oder Byzanz? Interpretationsprobleme am Beispiel der goldenen Mantelschließe von Dunapataj. In: Thesaurus Avarorum. Régészeti tanulmányok Garam Éva tiszteletére - Archaeological Studies in Honour of Éva Garam. Ed.: T. Vida. Budapest 2012, 207-224. 


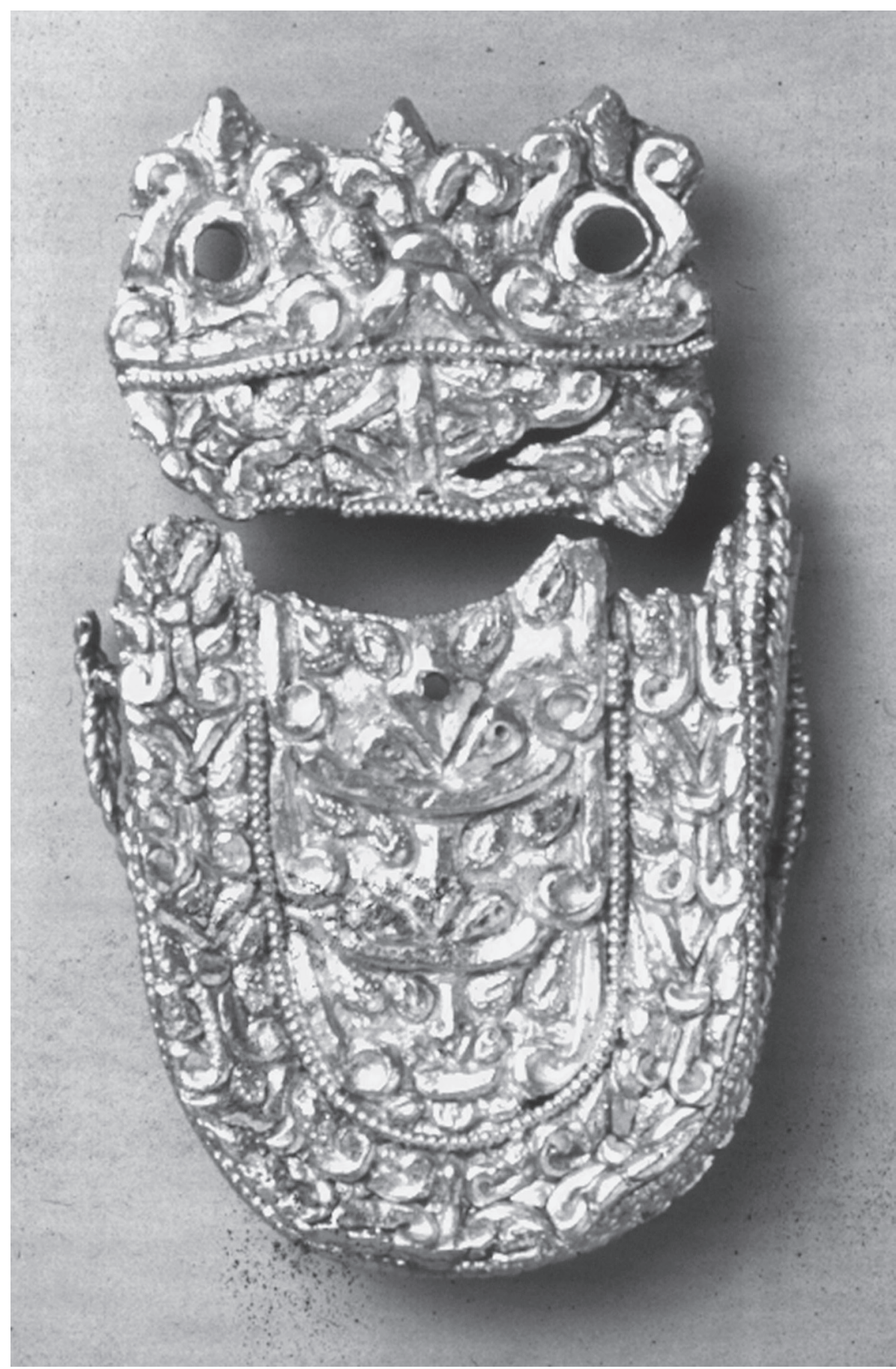

Abb. 3. Kunsthistorisches Museum Wien, Antikensammlung, Inv. Nr. VII B 79, 80 und 82. Goldene Hauptriemenzunge aus dem sog. „Schatzfund von Brestovac“, Kroatien.

Treibziselierte „Stäbchenrankenzier“ und tropfenförmige Blättchen der Varianten 2, 4b \& 5 (Foto: Institut für Ur- und Frühgeschichte, Universität Wien, Nicola Sautner) 


\section{FALLBEISPIEL 2: TROPFENFÖRMIGE BLÄTTCHEN}

Tropfenförmige Blättchen sind ein gebräuchliches und weit verbreitetes Zierelement auf frühbyzantinischen Feinschmiedearbeiten des 6.-7. Jahrhunderts (aus sowohl Edelmetall- als auch Kupferlegierungen) mit pflanzlichem Dekor. Allerdings sind hier lediglich zwei Varianten belegt:

- Variante 1: Tropfenförmige Blättchen mit zentraler, tropfenförmiger Kerbe

- Variante 2: Tropfenförmige Blättchen mit Punkt-Komma-Zier

Auch auf ,,italo-byzantinischen“ Goldschmiedearbeiten des 7. Jahrhunderts mit pflanzlichem Dekor kommen beide Varianten vor, fallweise sogar gemeinsam, wie z. B. als Komponenten der Halbpalmettenzier auf dem Goldblattblattkreuz von Stabio ${ }^{28}$.

In der pflanzlichen Ornamentik des Schatzfundes von Nagyszentmiklós ist das Zierelement ,tropfenförmiges Blättchen“ in fünf Varianten vertreten, die sich bezüglich der Binnenstrukturierung folgendermaßen unterscheiden:

- Variante 1: Tropfenförmige Blättchen mit zentraler, tropfenförmiger Kerbe (Nagyszentmiklós Gefäß-Nr. 7, 9 und 10, nach Hampel)

- Variante 2: Tropfenförmige Blättchen mit Punkt-Komma-Zier (Nagyszentmiklós Gefäß-Nr. 8, 13, 14, 15, 16, 19, 20 und 21, nach Hampel; vgl. Abb. 2)

- Variante 3: Tropfenförmige Blättchen ohne Binnenstrukturierung (Nagyszentmiklós Gefäß-Nr. 6, 7, 8, nach Hampel; vgl. Abb. 1)

- Variante 4: Tropfenförmige Blättchen mit zentraler Kerbe und feiner, flächendeckender Punktierung (Nagyszentmiklós Gefäß-Nr. 13, 14, 15, 16, 20 und 21 (nach Hampel)

- Variante 4a: Zentrale Kerbe mittels eines länglichen, tropfenförmigen Musterpunzens (= eindeutig tropfenförmige Form der zentralen Kerbe) gestaltet (Nagyszentmiklós Gefäß-Nr. 13, 14, 20 und 21, nach Hampel; vgl. Abb. 2)

- Variante 4b: Zentrale Kerbe mittels eines Schrotmeißels (= unregelmäßig gestaltete zentrale Kerbe) gestaltet (Nagyszentmiklós Gefäß-Nr., nach Hampel: 15, 16 und 20)

- Variante 5: Tropfenförmige Blättchen mit feiner, flächendeckender Punktierung, aber ohne zentrale Kerbe (Nagyszentmiklós Gefäß-Nr., nach Hampel: 13, 14, 20 und 21)

Bemerkenswert ist, dass die - auf byzantinischen Goldschmiedearbeiten gebräuchliche - Variante 1 (= tropfenförmige Blättchen mit zentraler, tropfenförmiger Kerbe) im Schatzfund von Nagyszentmiklós nur auf Gefäßen auftritt, die noch ins 7. Jahrhundert datieren dürften. Es handelt sich hierbei um das Schalenpaar Nr. 9 und 10 sowie den Krug Nr. 7. Interessant ist in diesem Zusammenhang auch, dass die aus tropfenförmigen Blättchen zusammengesetzten Motive - achtblättrige Rosetten auf dem Krug Nr. 7 bzw. Dreiblattmotive als Teil eines Kreuzmotivs beim Schalenpaar Nr. 9 und 10 - gute Parallelen in der italo-byzantinischen Kunst besitzen. Nach Cs. Bálint $^{29}$ ist das Zierelement „,tropfenförmige Blättchen mit tropfenförmiger Kerbe“ ein wichtiger Hinweis, der für die Datierung dieser drei Gefäße aus dem Schatzfund von Nagyszentmiklos (Nr. 7, 9 und 10 nach Hampel) ins 7. Jahrhundert sprechen könnte.

Im Gegensatz dazu sind die Varianten 4 und 5 ausschließlich auf jenen Gefäßen des Schatzfundes von Nagyszentmiklós belegt, die der „Stäbchenrankengruppe“ angehören und daher (zumindest) ins fortgeschrittene 8. Jahrhundert zu stellen sind. Tropfenförmige Blättchen der Varianten 4 und 5 sind auch auf dem ebenfalls mit Stäbchenranken- bzw. Lilienzier versehenen, treibziselierten goldenen Gürtelschmuck von Brestovac (Abb. 3) und Mátészalka $(A b b .4)$ belegt, was für eine Herstellung in eng verwandten Werkstätten sprechen dürfte ${ }^{30}$. Die flächige Punktierung mittels feiner Perl- oder Körnerpunzen findet sich auf zahlreichen Gefäßen aus dem Schatzfund von Nagyszentmiklós. Insgesamt wird diese Technik auf Gefäßen des Schatzfundes sowohl zur Binnenstrukturierung treibziselierter Tierkörper als auch tropfenförmiger Blattmotive (mit oder ohne zentrale Kerbe) verwendet.

Sowohl in herstellungstechnischer als auch in stilistischer Hinsicht sind Übereinstimmungen zwischen einer Gruppe italo-byzantinischer Feinschmiedearbeiten mit Halbpalmettenzier ${ }^{31}$ und der qualitativ hochwertigsten

\footnotetext{
${ }^{28}$ A. PERONI: L'arte nell'età longobarda. Una traccia. In: Magistra barbaritas : i barbari in Italia. A cura di G. Pugliese Carratelli. Antica madre: collana di studi sull'Italia antica 7. Milano 1986 , Tav. VI/1.
}

\footnotetext{
${ }^{29}$ BÁLINT 2010, 495-497.

${ }^{30}$ BÜHLER 2014, 159-161.

${ }^{31}$ RICCI 2012, 1-16.
} 


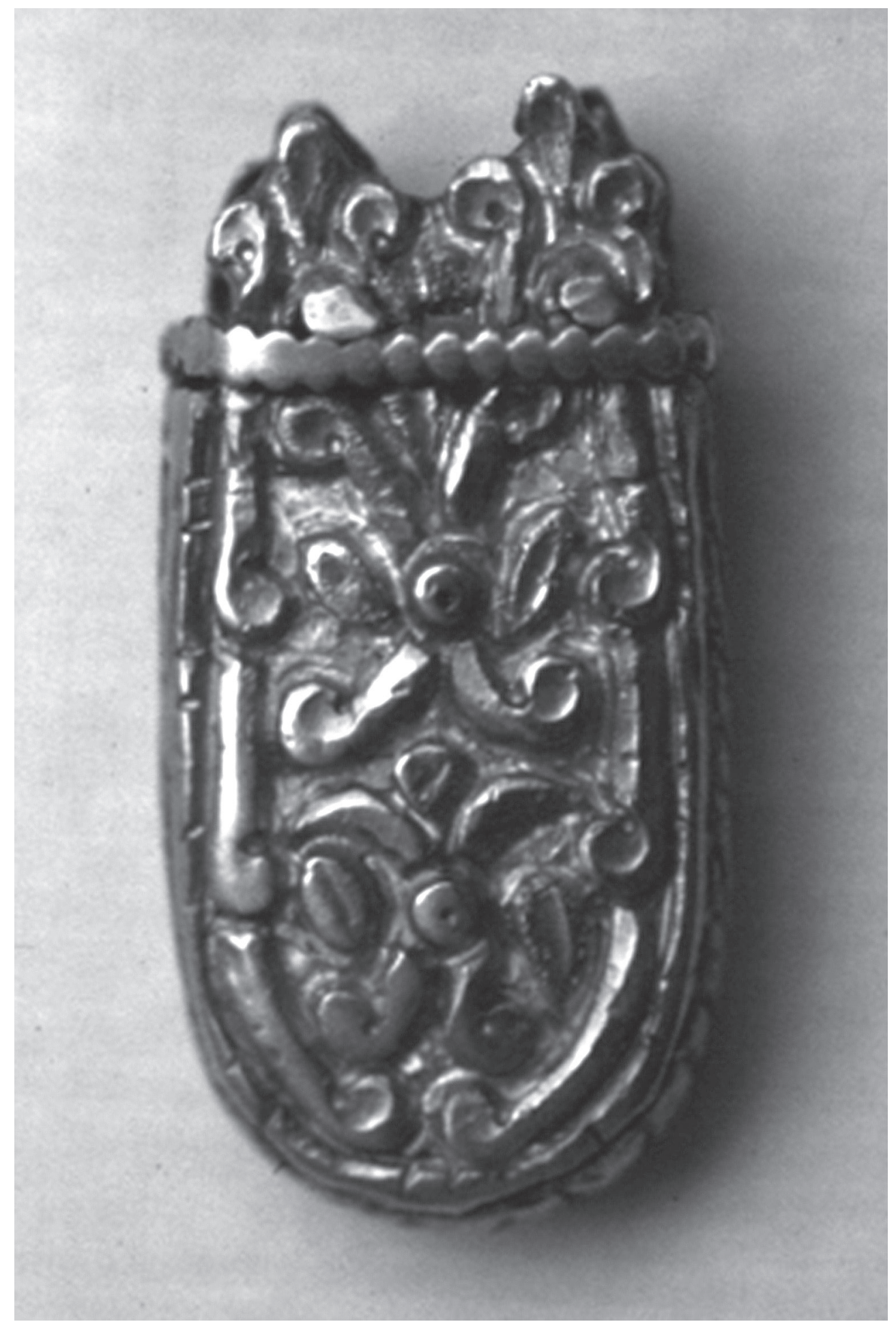

Abb. 4. Ungarisches Nationalmuseum, Budapest, Inv. Nr. 137/1909.

Goldene Nebenriemenzunge aus Mátészalka, Ungarn. Treibziselierte „Lilienzier“ und tropfenförmige Blättchen der Varianten $4 \mathrm{~b} \& 5$ (Foto: Falko Daim) 
Treibziselierarbeiten des 7. und 8. Jahrhunderts aus dem awarischen Siedlungsgebiet ${ }^{32}$ erkennbar. Besonders zu beachten ist in diesem Zusammenhang die Strukturierung von Flächen im Bereich des treibziselierten Dekors durch flächendeckende Punktierung mittels feiner Perl- oder Körnerpunzen: So wurden beispielsweise auf dem Sattelbeschlag aus Castel Trosino Grab 119 die Körper der beiden großen, geflügelten Tiermotive am linken bzw. rechten Rand durch flächendeckende Punktierung mittels eines auffallend feinen Perl- oder Körnerpunzens gestaltet.

Angesichts der hier diskutierten Übereinstimmungen wäre es denkbar, dass zumindest ein Teil des Goldschatzes von Nagyszentmiklós von Goldschmieden hergestellt worden ist, die in italo-byzantinischen Werkstätten ausgebildet worden sind.

\section{LITERATUR}

BÁLINT 2010

Brogiolo-ChaVARRÍA ARNAU 2007

BÜHLER-FREIBERGER 2018

BÜHLER 2014

GARAM 2002

RICCI 2012

SCHULZE-DÖRRLAMM 2009
= Cs. BÁLINT: Der Schatz von Nagyszentmiklós. Archäologische Studien zur frühmittelalterlichen Metallgefässkunst des Orients, Byzanz' und der Steppe. VAH 16b. Budapest 2010.

$=$ G. P. BRogiolo-A. ChavarRíA ARnAU (a cura di): I Longobardi. Dalla caduta dell'Impero all'alba dell'Italia. Catalogo della mostra, Torino-Novalesa, 2007-2008. Milano 2007.

$=$ B. BÜHLER-V. FREIBERGER: Die Ergebnisse der goldschmiedetechnischen und chemischen Untersuchungen. In: Der Goldschatz von Sânnicolau Mare (ungarisch: Nagyszentmiklós). Hrsg.: F. Daim, K. Gschwantler, G. Plattner, P. Stadler. Monographien des RGZM 142. Mainz 2018.

= B. BÜHLER: Der „Schatz“ von Brestovac, Kroatien. Seine kulturelle Beziehungen und technologischen Aspekte. Monographien des RGZM 85. Mainz 2014.

= É. GARAM: Die Verbindung awarenzeitlicher Fürsten- und Gemeinvolk-Grabfunde mit dem Schatz von Nagyszentmiklós. In: Gold der Awaren. Der Goldschatz von Nagyszentmiklós. Eine Ausstellung des Magyar Nemzeti Múzeum, Budapest, 24. März bis 30. Juni 2002. Hrsg.: T. Kovács. Budapest 2002 .

$=$ M. RICCI: Rome-Byzantium affinity and difference in the production of luxury goods. In: Byzantine Small Finds in Archaeological Contexts. Eds: B. Böhlendorf-Arslan, A. Ricci. BYZAS 15. Istanbul 2012.

= M. SCHULZE-DöRRLAMM: Byzantinische Gürtelschnallen und Gürtelbeschläge im Römisch-Germanischen Zentralmuseum. 2. Kataloge Vor- und Frühgeschichtlicher Altertümer 30/2. Mainz 2009. 
